\title{
Embedded System based Bangla Intelligent Social Virtual Robot with Sentiment Analysis
}

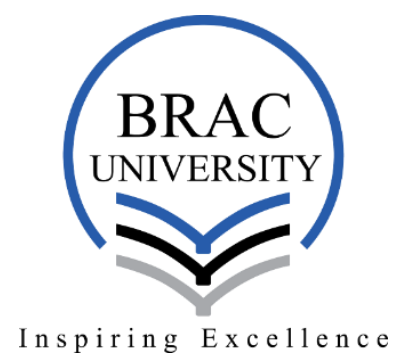

SUBMISSION DATE: 25.03.18

SUBMITTED BY:

Md. Ishan Arefin Hossain (14301032)

Ismail Hossain (14301133)

Md. Yousuf Hossain (14301136)

Mridul Banik (14301064)

Department of Computer Science and Engineering

Supervisor:

Amitabha Chakrabarty, Ph.D

Assistant Professor

Department of Computer Science and Engineering 


\section{Declaration}

We, hereby declare that this thesis is based on results we have found ourselves. Materials of work from researchers conducted by others are mentioned in references.

Department of Computer Science and Engineering 


\section{ABSTRACT}

Bangla is the mother tongue of millions of people all over the world. Despite of being a very popular language, any social virtual robot that can intelligently communicate in Bangla is a fairytale till now. One of the main reason of this is lack of rich text corpus and previous research on Bangla language. The proposed Bangla Intelligent Social Virtual Robot can communicate in Bangla intelligently and can express its reflective emotion virtually with the help of machine learning algorithms and sentiment analysis. In this paper we discuss the approached system, design methodology and implementation details of first ever Bangla virtual embedded robot followed by the methodology of building a rich Bangla text corpus. The proposed embedded virtual robot turns out better performer when compared with only known Bangla intelligent chatbot named 'Golpo' and the embedded system performance efficiency has been upgraded with the help CPU over-clocking technique 


\section{ACKNOWLEDGEMENT}

We would like to express our sincere gratitude to The Most Merciful Allah who blessed us with patience, determination, ability and opportunity to complete the research work within time.

We would love to thank from the core of our heart to our supervisor Dr. Amitabha Chakrabarty who has accepted us to do thesis under his supervision and has helped us to complete it within time with his valuable guidance and advises. We are fortunate and grateful to be able to work under his supervision.

We are grateful to the online based group "Vasha Shainik" who have helped us translating English subtitles and providing all of their work till now in order to build the first ever Bangla text corpus. Without these dedicated, hardworking and patriot soul, the long cherished Bangla Corpus still would have remained a dream. Moreover, we are under obligation of Dr. Ashraful Alam for providing us utmost support and his credit card to that helped us bearing the expenses of this virtual robot.

Finally, we are grateful to all of our family members and faculty members of Department of Computer Science and Engineering, BRAC University for believing in our ability and for supporting and encouraging us through thick and thin. 


\section{TABLE OF CONTENTS}

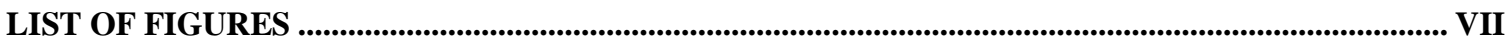

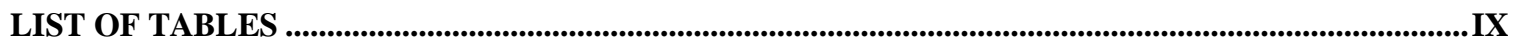

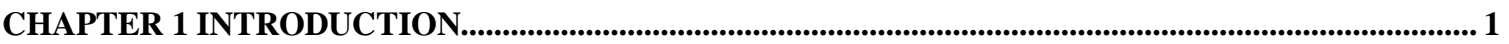

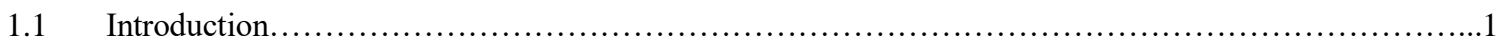

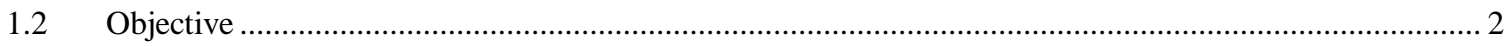

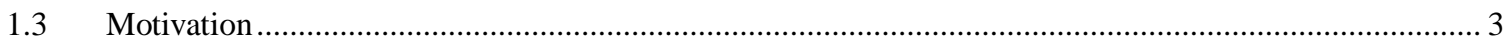

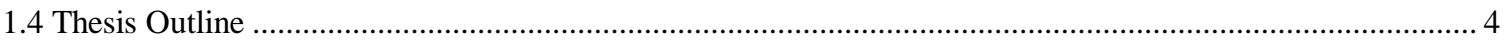

CHAPTER 2 LITERATURE REVIEW

CHAPTER 3 DATA COLLECTION AND REFINING PROCESS...................................................................... 8

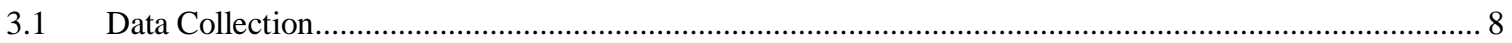

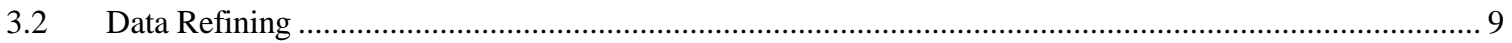

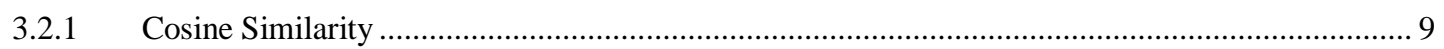

3.2.2 Classification of Bangla Sentence …………………............................................................ 14

CHAPTER 4 METHODOLOGY AND SYSEM PROCESS .......................................................................... 20

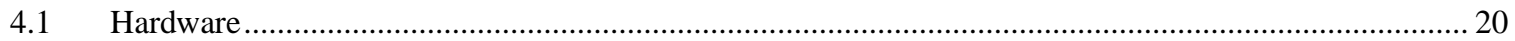

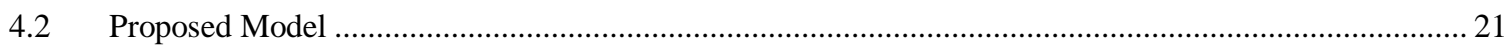

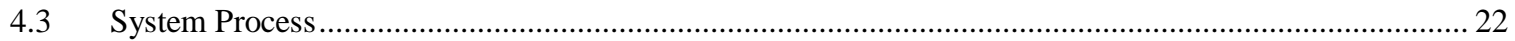

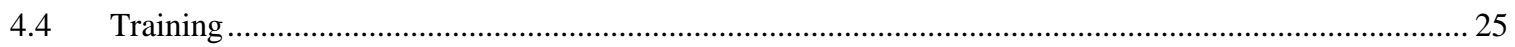

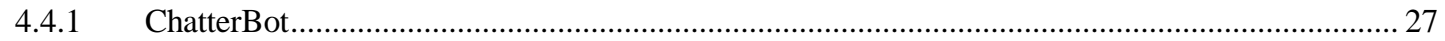

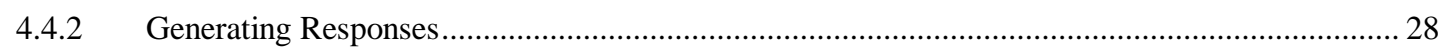

4.4.3 Best Match Logic Adapter ................................................................................................... 30

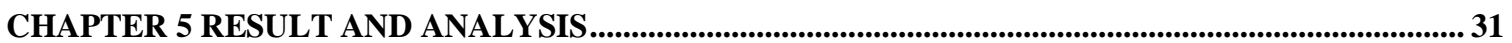

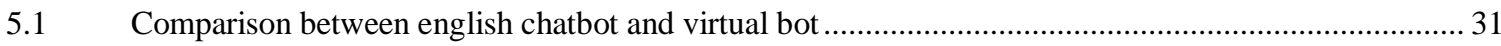

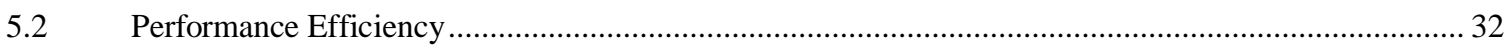

CHAPTER 6 CONCLUSION AND FUTURE WORK........................................................................................35

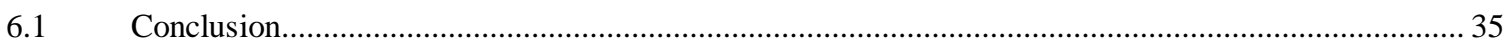

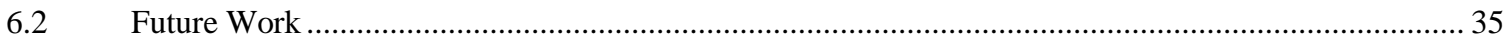

\section{REFERENCES}




\section{LIST OF FIGURES}

\begin{tabular}{|l|l|l|l|}
\hline $\begin{array}{l}\text { SERIAL } \\
\text { NO. }\end{array}$ & $\begin{array}{l}\text { FIGURE } \\
\text { NO. }\end{array}$ & \multicolumn{1}{|l|}{ NAME } & PAGE NO. \\
\hline 1 & 3.1 .11 & Unrefined data & 8 \\
\hline 1 & 3.2 .12 & Cosine graph of 2 sentence & 9 \\
\hline 2 & 3.2 .13 & Cosine Similarity Work Process & 11 \\
\hline 3 & 3.2 .14 & Intersection of A and B sets & 12 \\
\hline 4 & 3.2 .15 & Dataset after cosine similarity & 13 \\
\hline 5 & 3.2 .21 & Workflow of Naive Bayes classifier & 15 \\
\hline 7 & 3.2 .24 & $\begin{array}{l}\text { Result and performance of Naïve Bayes } \\
\text { Classifier }\end{array}$ & 18 \\
\hline 6 & 3.2 .22 & Train set sample & 16 \\
\hline & & & Test set sample \\
\hline
\end{tabular}




\begin{tabular}{|l|l|l|l|}
\hline $\begin{array}{l}\text { SERIAL } \\
\text { NO. }\end{array}$ & $\begin{array}{l}\text { FIGURE } \\
\text { NO. }\end{array}$ & \multicolumn{1}{|c|}{ NAME } & PAGE NO. \\
\hline 9 & 3.2 .25 & Final Refined Data & 19 \\
\hline 10 & 4.21 & $\begin{array}{l}\text { Schematic View of Bangla Social Virtual } \\
\text { Bot }\end{array}$ & 22 \\
\hline 11 & 4.31 & $\begin{array}{l}\text { Working Principle of Bangla Social Virtual } \\
\text { Bot }\end{array}$ & 24 \\
\hline 12 & 4.41 & $\begin{array}{l}\text { Visual representation of graph stored in } \\
\text { database }\end{array}$ & 26 \\
\hline 13 & 4.4 .21 & $\begin{array}{l}\text { Working Flowchart of } \\
\text { ChatterBot API }\end{array}$ & 29 \\
\hline 14 & 5.11 & $\begin{array}{l}\text { Comparison between a Chatbot \& } \\
\text { the Virtual Bot }\end{array}$ & 32 \\
\hline 15 & 5.21 & \begin{tabular}{l} 
Consumption time vs. CPU speed \\
\hline
\end{tabular} & 33 \\
\hline
\end{tabular}




\section{LIST OF TABLES}

\begin{tabular}{|l|l|l|l|}
\hline $\begin{array}{l}\text { SERIAL } \\
\text { NO. }\end{array}$ & $\begin{array}{l}\text { TABLE } \\
\text { NO. }\end{array}$ & NAME & PAGE NO. \\
\hline 1 & 3.2 .21 & Frequency Table of training set & 17 \\
\hline 2 & 5.21 & $\begin{array}{l}\text { Comparison between CPU speed and } \\
\text { time consumption }\end{array}$ & 33 \\
\hline 3 & 5.22 & Time consumption after threading & 34 \\
\hline
\end{tabular}




\section{CHAPTER I}

\section{INTRODUCTION}

\subsection{Introduction}

The ultimate objective of Artificial Intelligence, robotics is betterment of human life by making it easier with information, solve of problem and reducing the hazard of boring, time consuming jobs. From time to time, researchers have successfully reduced constrains of development of robotics by delivering robust solutions to major technical problems [5]. As a result, the amalgamation of robotics in our industrial and social life is becoming more and more mature. From a tele-operator to travel companion, implementation of robotics shines in every corner. Social robots in this case play a vital role for artificial intelligence. After the invention of the much loved hitchhiking robot named hitchBOT [3] the maker received a huge fund by crowdsourcing. When they wanted to send it out in the wild for an adventure, to their utter surprise, they observed many of the followers of the robot accompanied with it and realized how much impact one robot can make in social life [4]. Since then, researchers have put their heart and soul to bring new technologies and advancement of social robot. One of the best form of social robot is virtual robot since it is virtual robot not is bounded by any hardware restrictions and can deliver some outstanding emotion through its output devices like digital screens. There have been many advancement in virtual robot technology over the past decade. However, most of them are in English or Japanese language. There has been no initiative by any individuals or organization for development of a Bangla virtual robot except one close domain Chabot Golpo [2] despite the fact that Bangla is the 6th most spoken language (Bangla) across the world. On 
the other hand a fully functional prototype of a virtual wife robot is going to be launched by the end of 2018 and it has already earned mass popularity among consumers [1]. All these fact inspired us to take initiative for first ever Bangla social virtual robot.

However, to function in a social environment, a robot must acknowledge human being's social behavior [6]. To achieve that first and foremost condition for a robot is to understand natural language of the speaker first. One can call a robot intelligent enough for being a social robot if it can pass Turing test [4] in the communication sector.

Our virtual robot is packaged in an embedded system called raspberrypi. We have collected our dataset form number of sources then refined it in number of ways. After training the dataset in machine learning algorithm we have analyzed the sentiment of the robot. Our virtual robot changes its expression according to the sentiment we found through sentiment analysis.

\subsection{Objective}

1. To create a social virtual robot in Bengali which can understand users question and reply in Bangla naturally and not bounded by a specific domain.

2. To develop a Bengali corpus for training the virtual robot as well as the assistance of future research work.

3. To develop a Bengali corpus for training the virtual robot as well as the assistance of future research work.

4. The robot must change its emotions in the screen through sentiment analysis. 


\subsection{Motivation}

Most recent decade might have been those decade the place a technological revolution occurred. From scrutinizing in the example about customer conduct technique identified with smartphones we find the importance of having good virtual bot. With the goal the post- app time requests another pattern which might make be virtual robot. The user normally searches those results for their issues in Google, Yahoo, and other search engines anyway. Possibly they don't recover brief, alternately important information, or they recover documents alternately joins to these documents

Virtual bots can be new adaptation of the apps or the seek engines which will be able to collaborate with the user in accustomed language. It is getting apparent that the bound amount of apps in which the users accept bedfast them to are mostly amusing and messaging apps. So we can absolutely say it is an adumbration for the development of virtual robots. Age of interacting with computers with agreed commands or beat on the graphical user interface is continued, now it is the appeal of time the computer starts to yield commands in accustomed language.

With the advance of online-based casework like shopping, acclimation foods or any official works, it has become all-important to handle ample chump abject from all over the apple at any time. Recently abounding developments took abode in NLP analysis so with the ample availability of architecture it is the time to alteration to demography accustomed accent inputs interface. "The charge of communicative agents has become astute with the boundless use of claimed machines with the ambition to acquaint and the admiration of their makers to accommodate accustomed accent interfaces.. So all the abovementioned affidavit are the factors that played an affecting role which motivates us to body a virtual bot. 
With about 230 million speakers, Bangla is one of the large announced languages in the world, but little amount of linguistic accoutrement and assets for developing robots are accessible for it. For instance, there is no morphological analyzer, POS tagger or syntax parser accessible for Bangla. As a nation with a glorious history, Bengali is one of the civic language, the development of accent assets and its availability is a have to for acceptable Accent processing capabilities and analysis in this acreage .This actual angle

motivated us to build Bengali virtual bot. With the acceleration of amusing messaging apps users a part of Bengali people, it is the complete appeal of time to build a Bengali virtual bot.

\subsection{Thesis Outline}

Chapter One introduces the treatise of human-computer interaction, the need of chatbot and motivation behind this work.

Chapter Two discusses a brief history of chatbot and related works to our systems

Chapter Three explains the methodology, algorithm, flowchart, training and implementation of the prototype.

Chapter Four provides the sample of the experiments done on the systems, comparative studies of Golpo with other chatbot and analysis on it. 


\section{CHAPTER II}

\section{LITERATURE REVIEW}

We have made a social virtual robot which can talk in Bangla. There are many researches published regarding social virtual robot. However, most of them are developed in English language. We have found limited resources to develop our virtual bot. We have gone through a number research papers during our research. However, the major difficulty we faced is communication with user in Bangla. One of the early work of communication with user is chatbot, which can communicate with users contextually and naturally. Ironically, there is not even one successful implementation of general purpose chatbot. As a result we tried our best to create our own general purpose chatbot that is not limited to a specific domain.

In early ages chatbtots were dependent on handcraft templates or heuristic value to generate reply. In spite of requiring tremendous effort it could only generate few responses [15].However, recent researches are working on data driven approaches to build fully functional chatbots [16]. Statistical goaloriented dialogue systems have long been modeled partially. A paper recently applied deep reinforcement learning successfully to train non-goal oriented chatbot type dialogue agents. They have shown that reinforcement learning allows the agent to model long-term rewards and generate more diverse and coherent responses as compared to supervised learning [17]. 
Some work was based on the idea of web based voice chat bot using black box approach. In 2008 A paper called "Humorist Bot: Bringing Computational Humor in a Chat-Bot System" presented the idea of building bot with humor. The bot will also change its expression according to the humoristic content of the dialogue [18]. Virtual Conversation with a Real Talking Head" a paper demonstrated a bot which specifies with a system using an animated face model synchronized with a speech synthesis Module. It is used like a presentation layer of a conversational Agent which provides reply [19]. Here the textual reply is transformed into facial movements of a 3D face model whose lips and tongue movements are synchronized with the sound of the synthetic voice. With the integration of the chatbots and other technologies virtual robots have made their appearance in the world.

Our whole system is conducted on a microcomputer called RaspberryPi model B. Raspberry-pi is a pocket size microcomputer run on Linux operating system. It is equipped with $1 \mathrm{~GB}$ ram, a micro-SD card, a CPU of ARM Cortex-A53, 40 GPIO pins [20]. From our research we have found different machine learning algorithms can run on this microcomputer. A number of papers used python and raspberry pi for security or intruder detection where python library openCV is used for image analysis [21, 22].Raspberry can also be used for communication intensive works. It's used for cloud based works [23]. That drives us to make our virtual bot in raspberry-pi microcomputer. 
In recent time one Japan based organization Gatebox showed its strength by showcasing and releasing world's first virtual robot wife assistant's TVC to the world and many preorders have counted since then. However, author is not aware of any virtual robot by the time of writing this paper. There are very few robots that can communicate in Bangla but, they can generate response of very few specific questions. On the other hand, our work includes open domain reply of the chatbot. 


\section{CHAPTER III}

\section{DATA COLLECTION \& REFINING PROCESS}

Preparing a well refined and well-structured Bangla Corpus was a big challenge. The lacking of resources regarding Bangla corpus made the job harder. We collect the raw data from an English Movie Corpus. Then Cosine Similarity for translation and complexity classification was done for refining.

\subsection{Data Collection}

Data collection is the process of gathering and measuring information on variables of interest, in an established systematic fashion that enables one to answer stated research questions, test hypotheses, and evaluate outcomes. We collected our data Cornell movie dialog corpus.

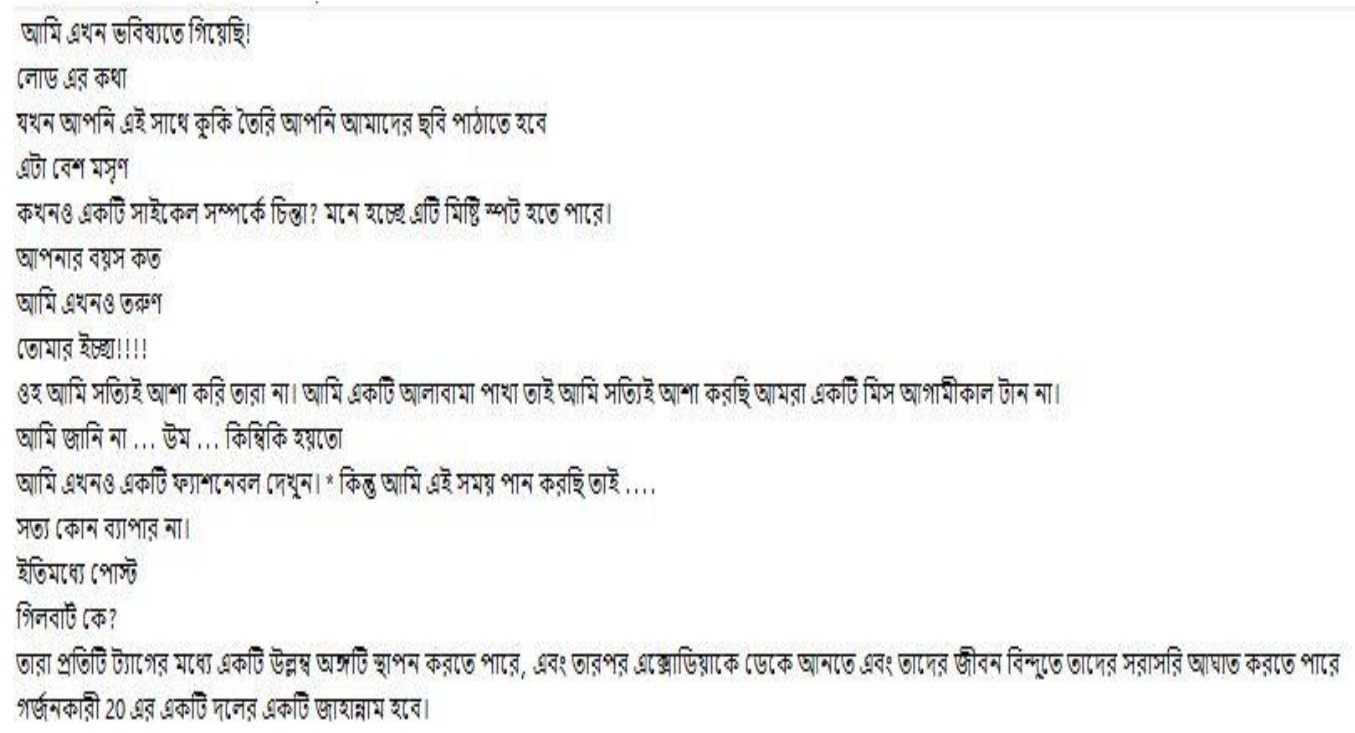

Figure 3.1 1: Unrefined Data 
This corpus contains a large metadata-rich collection of fictional conversations extracted from raw movie scripts. So, we had to refine this huge data to fit in our machine learning algorithm.

\subsection{Data Refining}

Data Refining is a process that refines disparate data within a common context to increase the awareness and understanding of the data, remove data variability and redundancy, and develop an integrated data resource [8].

\subsubsection{Cosine Similarity}

Our data refining process composed of many different subsets. One of them is to refine from translation. As we are translating a big portion of our data. For this, we are using Cosine Similarity, which is used to measure the similarity between two non-zero vectors. Cosine Similarity is very useful algorithm for machine learning. If, the cosine of $0^{\circ}$ is 1 , and it is less than 1 for any other angle in the interval $(0,2 \pi)[9]$.

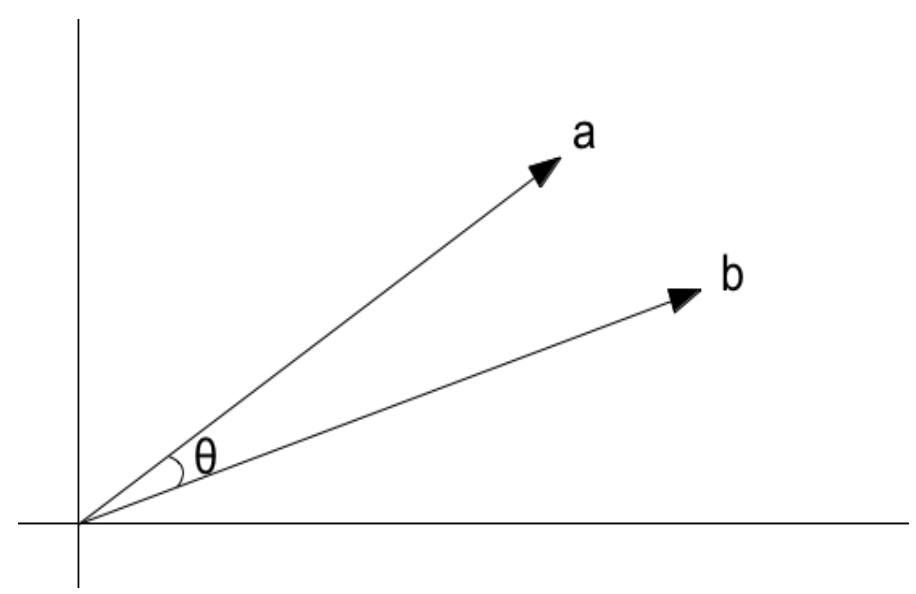

Figure: 3.2.1 2: Cosine graph of 2 sentence [12]. 
Here $\mathrm{a}$ and $\mathrm{b}$ are 2 sentence from our translated text. Two vectors with the same orientation have a cosine similarity of 1 , two vectors at $90^{\circ}$ have a similarity of 0 , and two vectors diametrically opposed have a similarity of -1 , independent of their magnitude. It shows if both translated text have the same sort of result, it's a good translation. Here the outcome is neatly bounded in $[0,1]$. Cosine distance is a term often used for the complement in positive space [10], that is:

$$
\begin{gathered}
\mathrm{Dc}(\mathrm{A}, \mathrm{B})=1-\mathrm{Sc}(\mathrm{A}, \mathrm{B})[9] \\
\mathrm{Dc}=\text { Cosine Distance, } \quad \mathrm{Sc}(A, B)=\text { Cosine Similarity }
\end{gathered}
$$

Here $\mathrm{D} c$ is the cosine distance and Sc is the cosine similarity. However, that this is not a proper distance metric as it does not have the triangle inequality property. To repair the triangle inequality property while maintaining the same ordering, it is necessary to convert to angular distance [3]. Given two vectors of attributes, $\mathrm{A}$ and $\mathrm{B}$, the cosine similarity, $\operatorname{Cos}(\theta)$, is represented using a dot product and magnitude as

$$
\begin{gathered}
\text { Similarity }=\operatorname{Cos} \theta=\frac{A \cdot B}{|A||B|}=\frac{\sum_{i=1}^{n} A i \cdot B i}{\sqrt{\sum_{i=1}^{n} A i^{2} \sqrt{\sum_{i=1}^{n} B i^{2}}}}[10] \\
A i=\text { First Sentence, } \quad B i=\text { Second Sentence, } \\
\theta=\text { Degree Between two sentence }
\end{gathered}
$$

Here $A i$ and $B i$ are components of vector $\mathrm{A}$ and $\mathrm{B}$ respectively. Here $\mathrm{A}$ and $\mathrm{B}$ are 2 vector of our sentence or text. The resulting similarity ranges from -1 meaning exactly opposite, to 1 meaning exactly the same, with 0 indicating orthogonality (decorrelation), and in-between values indicating intermediate similarity or dissimilarity. For text matching, the attribute vectors A and B are usually the term frequency vectors of the documents. The cosine similarity can be seen as a method 
of normalizing document length during comparison. In the case of information retrieval, the cosine similarity of two documents will range from 0 to 1 , since the term frequencies cannot be negative. The angle between two term frequency vectors cannot be greater than $90^{\circ}$. The following figure shows how our Cosine Similarity actually

works.

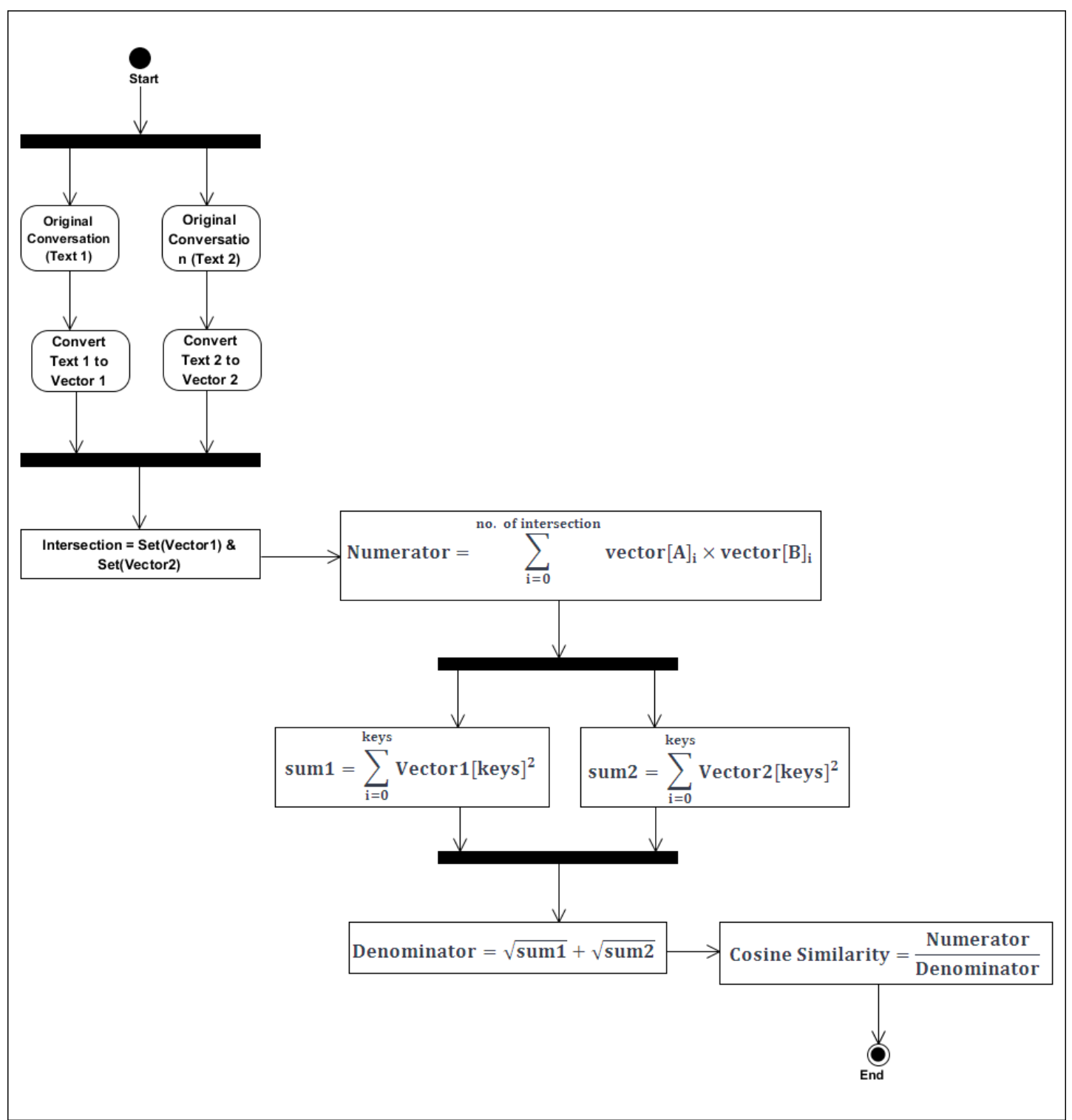

Figure: 3.2.1 3: Cosine Similarity Work Process 
First of all we need to need to refine the 1000 movie dataset by selecting dialog serial. Then, we used Google Cloud API to translate them into. Again we translate the Bangla conversation into English conversation to start our Cosine Similarity. We convert those 2 English text into vectors. These vectors have 2 components for all the word. One is the word itself and second is term frequency of the text. Term frequency is a numerical statistic that is intended to reflect

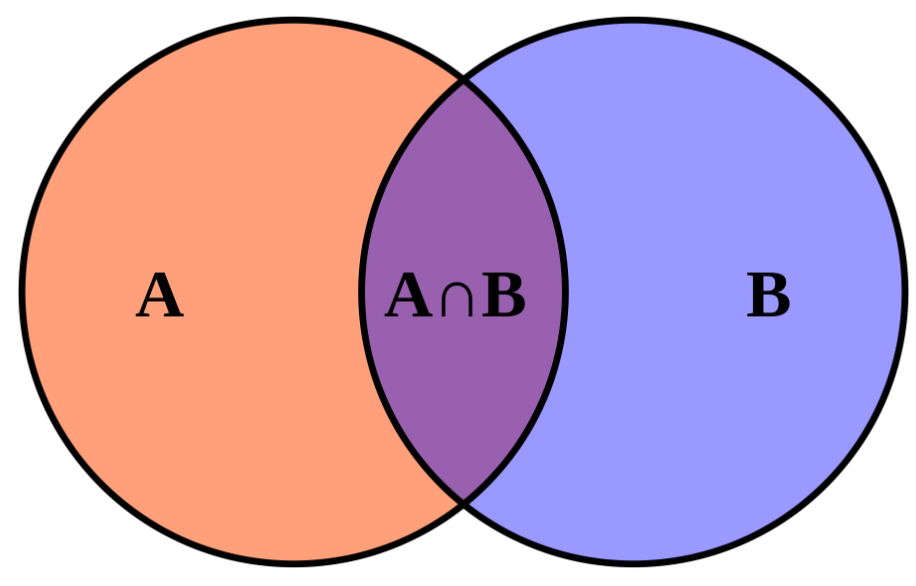

Figure: 3.2.1 4: Intersection of $A$ and $B$ sets [13]

Then for all these intersection words, we need to calculate

$$
\begin{aligned}
& \text { Numerator }=\sum_{i=0}^{n o \text { of intersection }} \text { vector } 1(A i) \times \operatorname{Vector} 2(\mathrm{~B} i)[10] \\
& \text { Denominator }=\sqrt{ } \sum_{i=1}^{n} A i^{2} \cdot \sqrt{ } \sum_{i=1}^{n} B i^{2}[10]
\end{aligned}
$$$$
A i=\text { First Senstence Vector } 1, \quad B i=\text { Second Sentence Vector } 2
$$ 
These Numerator is the dot product of the 2 vector A and vector B.

$\mathrm{A} \cdot \mathrm{B}=A x \cdot B x+A y \cdot B y+A z \cdot B z[11]$

$A$ is defined as $A x, A y, A z$ and $B$ is defined as $B x, B y, B z$

Cosine Similarity $(\operatorname{Cos} \theta)=\frac{\text { Numerator }}{\text { Denominator }}[10]$

So that's our Cosine similarity to find out the similarity between 2 translations and refine our dataset.

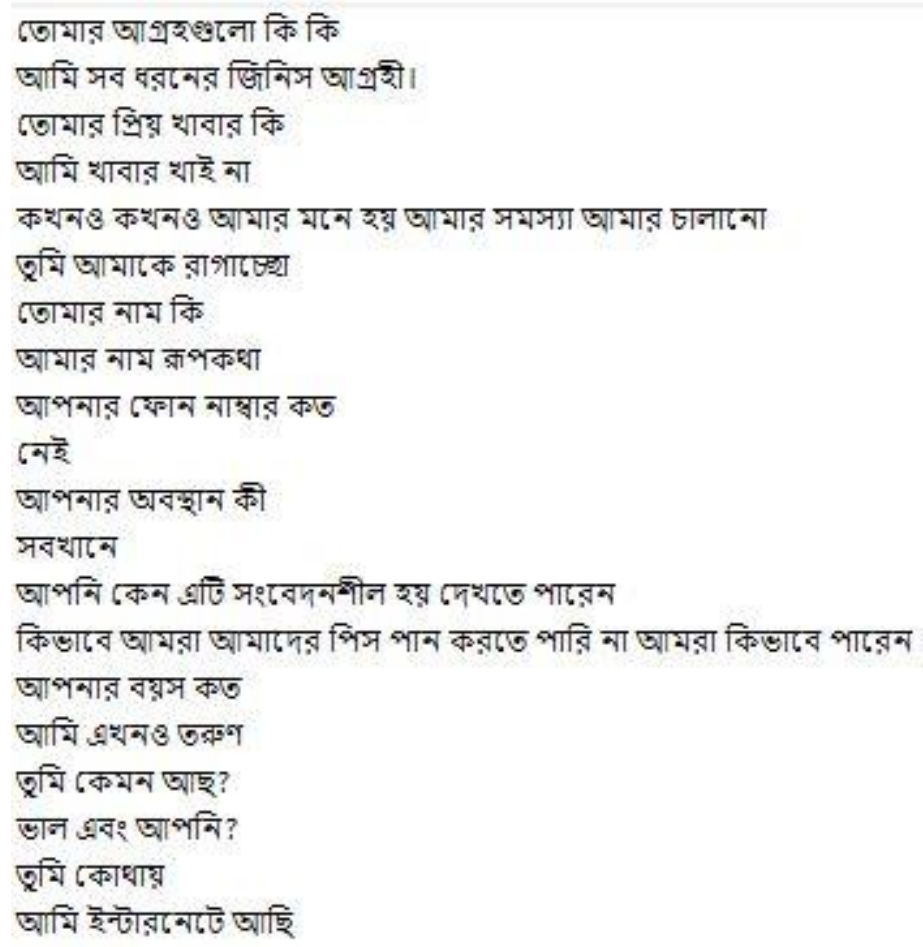

Figure: 3.2.1 5: Dataset after cosine similarity 


\subsubsection{Classification of Bangla Sentence}

After successfully translated and rechecked by crowdsourcing, another problem arose. We had to differentiate out three different type of sentence- সরল, য৮•গগকি, জটীল, which stands for simple, complex and compound sentence in English as most of the conversation uses simple sentence and simple reply will leave users joyful. One of the approach to differentiate these three type of sentence is clause identification [8] which gives merely satisfactory result with an average success rate of $70 \%$. The problem regarding clause identification is to counting verbs of the sentence and for that we need rich corpus of verbs. However, a language like Bangla can have many representation of one single English word which makes it unreliable for classification. For instance, one English verb "going” can have 5 meaning in Bangla such as যাচ্ছর, যাচ্ছ,, যাচ্ছ, যাইতছে, যাইতছে, যাইতছেরি

To resolve this particular issue, we propose a more mathematical approach like Naïve Bayes classification. It is a classification technique based on Bayes' Theorem with an assumption of independence among predictors. In simple terms, a Naive Bayes classifier assumes that the presence of a particular feature in a class is unrelated to the presence of any other feature. For example, a fruit may be considered to be an apple if it is red, round, and about 3 inches in diameter. Even if these features depend on each other or upon the existence of the other features, all of these properties independently contribute to the probability that this fruit is an apple and that is why it is known as 'Naive'.

Naive Bayes model is easy to build and particularly useful for very large data sets. Along with simplicity, Naive Bayes is known to outperform even highly sophisticated classification methods. 
Bayes theorem provides a way of calculating posterior probability $\mathrm{P}(\mathrm{c} \mid \mathrm{x})$ from $\mathrm{P}(\mathrm{c}), \mathrm{P}(\mathrm{x})$ and $\mathrm{P}(\mathrm{x} \mid \mathrm{c})$. Look at the equation below:

$$
P(c \mid x)=\frac{P(x \mid c) P(c)}{P(x)}
$$

$P(c \mid x)$ is the posterior probability of class (c, target)

given predictor (x, attributes).

$P(c)$ is the prior probability of class.

$P(x \mid c)$ is the likelihood which is the probability of predictor given class.

$P(x)$ is the prior probability of predictor.

A simple representation of Naïve Bayes looks like this

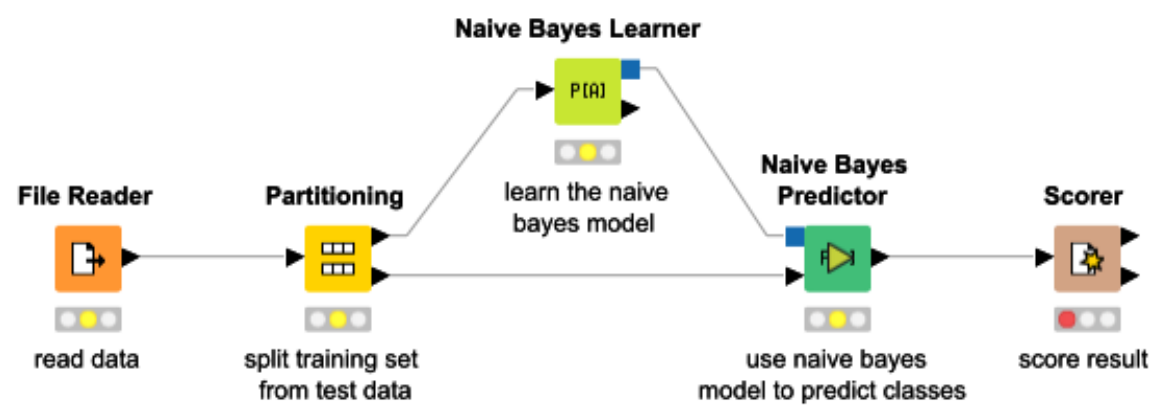

Figure: 3.2.2 1: Workflow of Naïve Bayes classifier [29]

For our case, we have created a training set of 300 simple, and 150 of complex and compound sentences each. We then labeled the simple sentences as "সরল" which stands for simple in Bangla and complex and compound sentences as 
“সরল নয়” which means the sentence is not simple in Bangla. Here is a partial representation of our training data.

('স্নেহময়া জননা ষ্বায় সন্তানকে প্রাণাপেক্ষা ভালোবাসেন', 'সরনল'), ('বিশ্ববিখ্যাত মহাকবিরা ঐন্দ্রজালিক শক্তিসম্পন্ন নেখনী দ্বারা অমরতার সঙ্গীত রচনা করেন।', 'সরনল '), ('পুকুরে পদ্ম ফেনটে।', 'সরনল'), ('ভালো ছেনেরা শিক্কের আদেশ পালন করে। সরূন বাক্য', 'সরূন'), ('তার দর্শনমাত্রই আমরা প্রস্থান করুলাম।', 'সরনল'),

('

('মाংসভোজ্জী পশু অত্যন্ত বনলান।', 'সরূন'),

('তিনি আমাকে পাঁ টাকা দিয়ে বাড়ি যেতে বলনেন।', 'সরুল'),

('পরীকায় উত্তীর্ণ হওয়ার জন্য এখন থেকেই তোমার পড়া উচিত।', 'সরূল '),

('আমি বহ্ট কট্টে শিক্ষা নাভ করেছি।', 'সরন '),

('সত্য কধ্ধা না বনে বিপদে পড়েছি।', 'সরুল '),

('তার বয়স হনেও বূদ্ধি হয় নি।', 'সরুন '),

('মেঘ গর্জন করেলে ময়র মৃত্য করে।', 'সরুল '),

|'যে পরিশ্রম করে , সেই সুখ নাভ্ড করে।', 'সরুল নয়'),

('সে যে অপরাধ করেছে তা মুখ দেখেই বুঝ্ছেছি।', 'সরুল নয়'),

('আমি মাঠে গিয়় দেখনাম, খেনা শেষ হয়ে গিয়েছে।।', 'সরূন নয় '),

('তিনি বাড়ি আছ্ছেন কিনা, আমি জানি না।', 'সরুল নয় '),

('খॉটি সোনার চাইতে খॉটি, আমার দেশের মাটি।', 'সরন নয়'),

('ধনধান্য পুচ্পে ভরা, আমাদের এই বসুন্ধ্রা।', 'সরুল নয় '),

('ঘে এ সভায় অনুপন্থিত সে বড় দুর্ভাগা।', 'সরনল নয়'),

('ত্মমি আসবে বলে আমি অপেক্ষা করছি।।', 'সরনল নয়'),

('যেখানে আকাশ আর সমূদ্র একাকার হয়ে গেছে সেখানেই দিকচক্রবাল।', 'সরনল নয়'),

('যদি সে কাল আসে, তাহলে আমি যাব।', 'সরূন নয়'),

('যখন বিপদ আসে, তখন দুঃখও আসে।', 'সরনল নয় '),

('যে পরিশ্রম করে,/ সে-ই সুখ নাভ করে।', 'সরনল নয়'),

\section{Figure: 3.2.2 2: Train set sample}

After training the dataset we then train our dataset with Naïve Bayes Classification algorithm. If we refer to the Bayes Theorem equation,

$$
P(c \mid x)=\frac{P(x \mid c) P(c)}{P(x)}
$$

Here, $\mathrm{c}$ indicates if a sentence is simple or not and $\mathrm{x}$ denotes a particular 
keyword. And $\mathrm{P}(\mathrm{c} \mid \mathrm{x})$ denotes probability of a sentence being simple if there is a particular word $\mathrm{x}$ is present in the sentence. The Naïve Bayesian classifier creates a frequency table and a likelihood table by finding the probabilities. Here is the visual Frequency distribution of our training set's most important keywords from which that determines whether a sentence is simple or not.

\begin{tabular}{|c|c|c|}
\hline Keyword & Simple & Not simple \\
\hline কिन्तु & 5 & 49 \\
\hline এবং & 7 & 57 \\
\hline যে & 11 & 68 \\
\hline যদি & 6 & 37 \\
\hline তथाপि & 2 & 11 \\
\hline ও & 13 & 3 \\
\hline उाল & 60 & 12 \\
\hline
\end{tabular}

Table 3.2.2 1: Frequency Table of training set

Next, we use Naive Bayesian equation to calculate the posterior probability for each class. The class with the highest posterior probability is the outcome of prediction. Finally, it is time to test the trained model, for which we have used the following test set. 


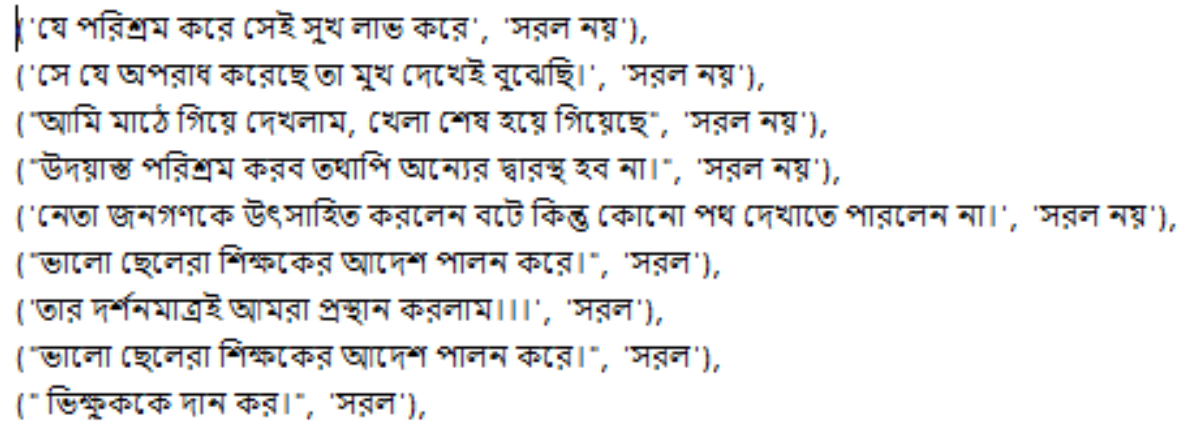

\section{Figure: 3.2.2 3: Test set sample}

The Naïve Bayes really shows it efficiency and effectiveness with an accuracy rate of $88.88 \%$. Moreover, when extracting features, the algorithm successfully pointed out the special words of compound and complex sentences. For example, in Bangla compound sentences usually contains “কিন্তু “, “এবং” , “অথবা” , “তথাপি” , “ও” keywords and complex sentences usually contains “যে”, ‘যারা”, ‘যত”,’যদি”, “সে”, ‘যিনি” [9]. We believe, more training set could lead us towards higher accuracy rate.

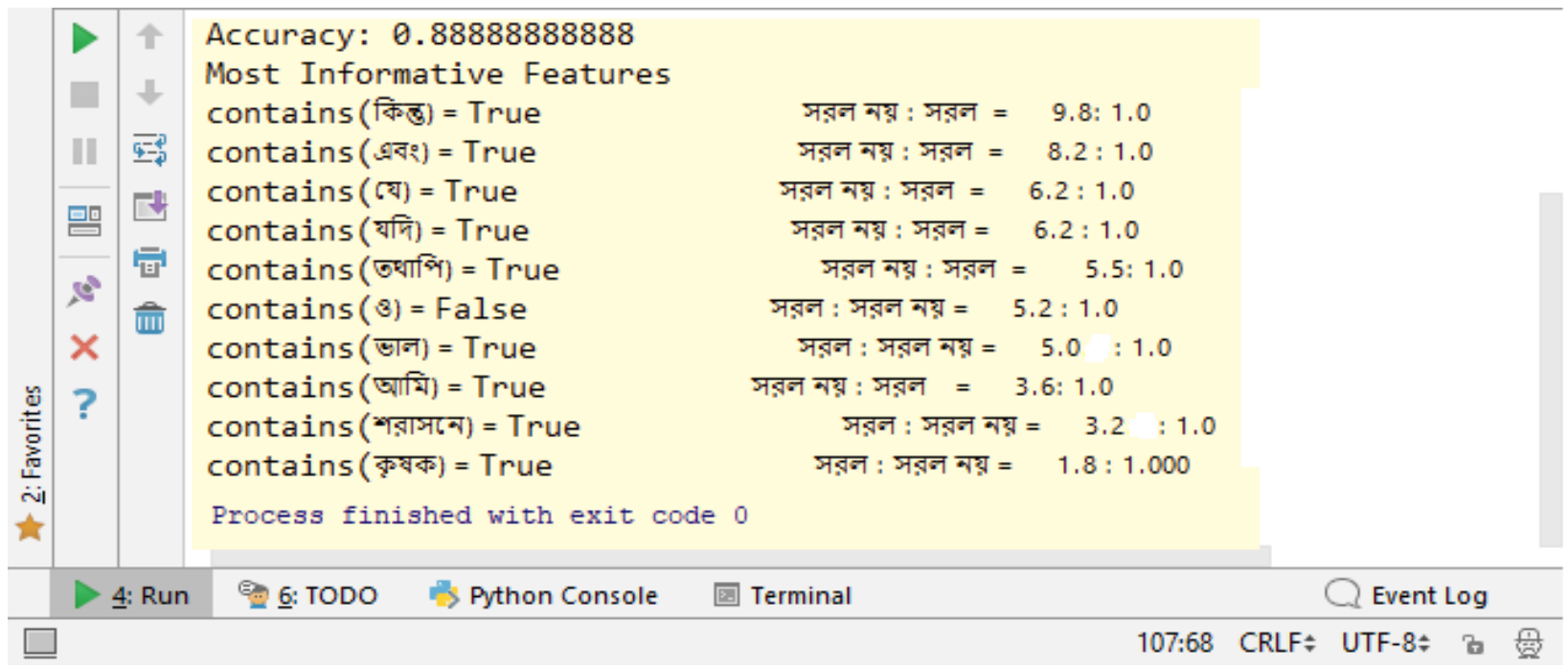

Figure: 3.2.2 4: Result and performance of Naïve Bayes Classifier 
'Finally, we get rid of the complex and compound sentences in our Bangla conversation corpus by the classifier we got from Naïve Bayes classification algorithm and achieved 25,682 conversation of simple sentences.

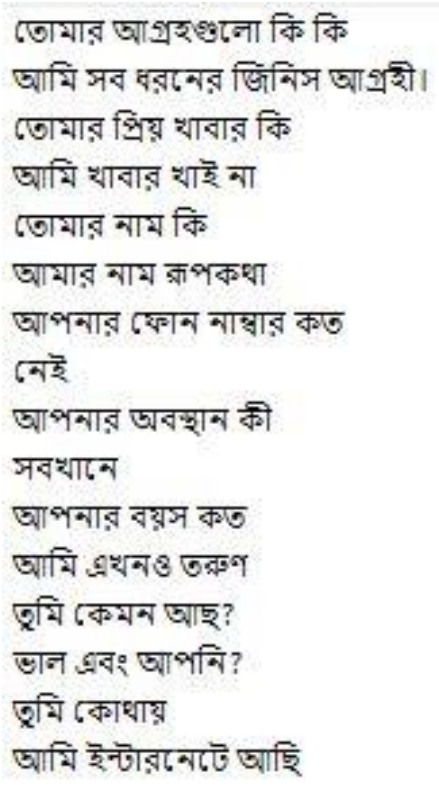

Figure: 3.2.2 5: Final Refined Data 


\section{CHAPTER IV}

\section{METHODOLOGY AND SYSTEM PROCESS}

The proposed model illuminates the architecture of the Bangla Virtual Bot Model which consist of Raspberry Pi and other hardware. The system process describes how the Bangla Virtual Bot initiates its output generating process from input.

\subsection{Hardware}

We have used some hardware to build our virtual bot. They are Raspberry-pi, microphone, usb-soundcard, display and speaker. By integrating all this hardware we build our bot. The architecture of our system is described below to depict the idea of the system.

\section{Raspberry-Pi:}

We have used Raspberry pi 3 model in our work [20]. Pi is a small computer with cheap price. It can be used by adding a keyboard, display, mouse, and possible some extra storage. Raspberry pi 3 contains Broadcom BCM2837 SoC. The CPU configuration is 4 X ARM Cortex-A53 where the clock speed is $1.2 \mathrm{GHz}$. It is also equipped with GPU. The version of the GPU is Broadcom VideoCore IV. It has also one GB ram which is LPDDR2 (900 $\mathrm{MHz}$ ). It has networking module named 10/100 Ethernet of $2.4 \mathrm{GHz} 802.11 \mathrm{n}$ wireless. The blue tooth module of this micro-computer is 4.1 classic .The storage mainly depends on micro SD. The memory of the storage depends on micro SD. This device has 40 pin header .Some of them are for $5 \mathrm{v}, 3 \mathrm{~V}$ and Ground, other pins are used as GPIO. This GPIO pins can be used as both input and output. Pi is equipped with number of ports. In ports it includes one HDMI 
port, one $3.5 \mathrm{~mm}$ analogue audio-video jack. It also come up with built in Ethernet .To drive camera and display pi provides Camera Serial Interface (CSI), Display Serial Interface (DSI).

\section{Other Components:}

Apart from raspberry-pi we have used microphone to take input from the user. Microphone actually has $2.5 \mathrm{~mm}$ jack which is not available in raspberry-pi. So we have used usb-soundcard to connect our microphone. We have used speaker to deliver the sound of response generated by our system. To visualize the sentiment of the input we used display which depicts whether the input was funny, neutral or sad. .

\subsection{Proposed Model}

The proposed working model of this virtual robot is based around the Raspberry Pi embedded system. The Raspberry pi is used to process the system processing algorithm of the virtual bot which is basically Machine Learning and Natural Language Processing. The method implements an LED Display as an I/O device to express the sentimental reflection of the bot virtually. It also implements a microphone as an I/O device in order to listen to human voice for bot's speech recognition. It implements speakers as I/O devices in order to get the verbal reflection of the virtual bot which generates through machine learning. 


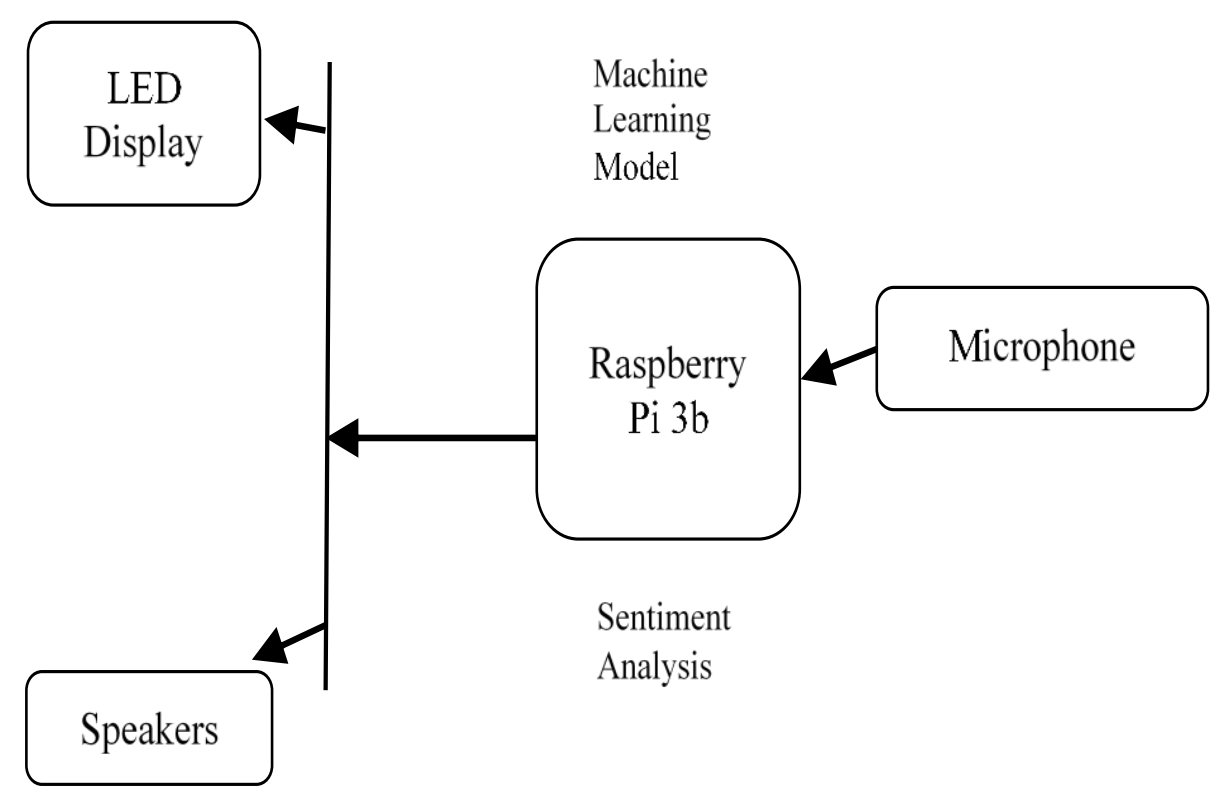

Figure 4.2 1: Schematic View of Bangla Social Virtual Bot

For triggering the virtual bot, a human have to get the microphone of the bot and ask the bot something in Bangla. The bot will then reply with the help of its machine learning model verbally and graphically. The verbal reply will be outputted from the speakers of the embedded system and the graphical sentimental reflection will be visible on the Raspberry Pi's LED Display.

\subsection{System Process}

For At first, the virtual bot detects human speech in Bangla. For detecting Bangla speech, Google's speech Application Programming Interface (API) is used here. The API manipulate through deep learning models in order to get the perfect speech recognition. Once the speech get detected, the system 
processing algorithm casts the speech value into a data frame object. Now, two operations will take place in different threads after this object casting.

Firstly, the data frame object then compared with training set by the Machine Learning AI in order to get the highest probable answer for that particular speech which can be a question or comment. Then the AI provides with the best probable answer in string form. The answer string then again feed into the Google Speech API. This time the API is used for the other way round. The answer string is turned to Bangla speech by the API with the help of deep learning. Phonetic voice triggered from the speakers of the embedded virtual bot sounds a man and the real human being who asked the question at the first place get the artificially intelligent answer from the virtual bot.

Simultaneously, the data from object of the speech feed into a sentiment analysis learning model. The model's Application Programming Interface is called TextBlob.

This model first translated the Bangla speech data frame object into an English text. Then the model implements part-of-speech tagging, noun phrase extraction some more operations by the help of common Natural Language Processing in order to get the sentiment of that particular speech. The return value of this sentiment analysis model differs from -1 to 1 .

-1: Uttermost disappointment/sadness

1: Uttermost joy/happiness 


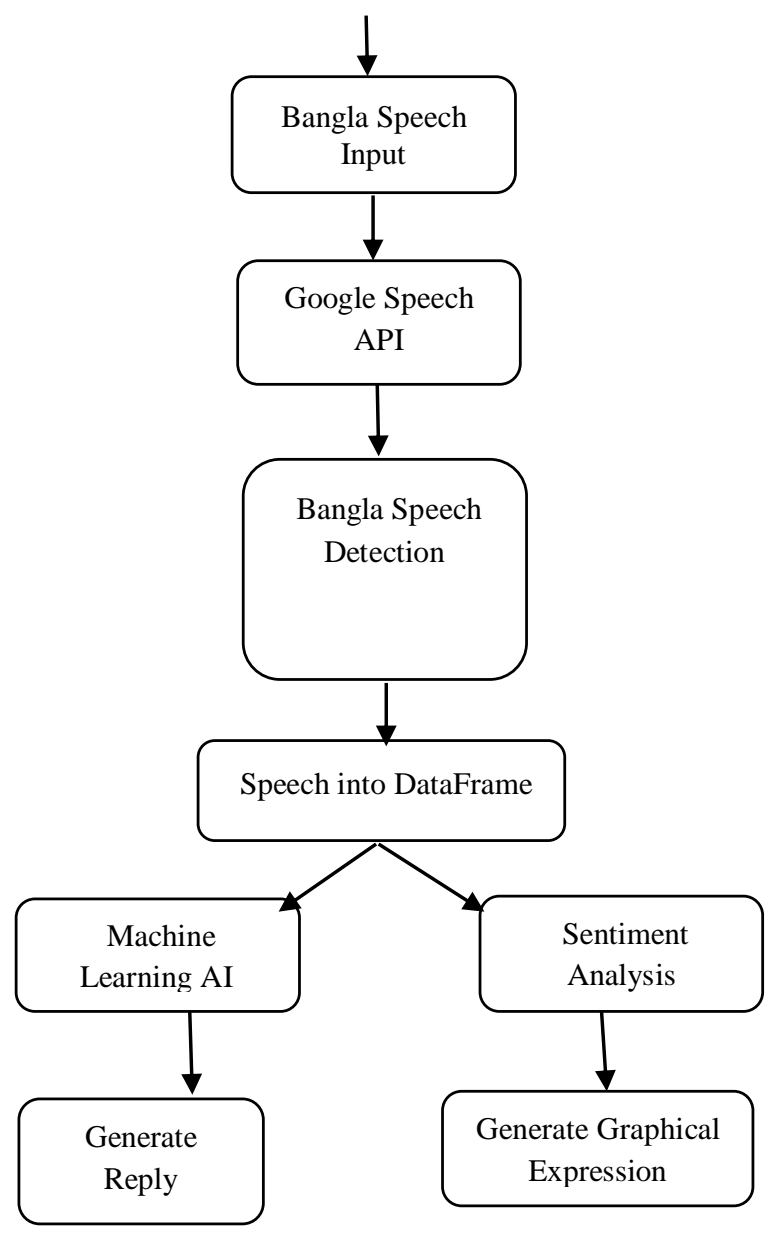

\section{Figure 4.3 1: Working Principle of Bangla Social Virtual Bot}

In between the value of -1 to 1 , it refers to more normal state or one can say the neural state of the speech. By judging the return value of the sentiment analysis, the system processing algorithm implements an Application Programming Interface of Python which is called Pillow in order to express the sentiment of the virtual bot in a graphical way so that the human being standing in front of the bot can understand its emotion from his/her committed comment or query. The virtual bot has three types of graphical sentimental reply on its virtual face. They are: 
Sad Face: Triggered for sentimental analysis return value from -1 to 0

Normal Face: Triggered for sentimental analysis return value from 0 to 0.3

Joyful Face: Triggered from sentimental analysis return value from 0.3 to 1

These two operations, generation intelligent answer and generating virtual graphical face based on sentiment give the virtual bot a social feel in front of its human companions.

\subsection{Training}

After refining our dataset we have trained our dataset. For training the dataset we have made data frame object and pass it into machine learning algorithm of ChatterBot. ChatterBot is a python library where various machine learning algorithms like decision tree algorithms, ansemble methods and clustering algorithms. One of the main advantage of these machine learning algorithms is that these are language independent which allows Chatterbot to be trained to speak any language.

The training process includes loading the dialogues from the corpus. This either creates or builds upon the graph data structure that represents the sets of known statements and responses. When a chat bot trainer is provided with a data set, it creates the necessary entries in the chat bot's knowledge graph so that the statement inputs and responses are correctly represented. However, one of the drawback of chatterbot training model is, it uses SQLlite database 
for training model. And while generating responses, it uses a search algorithm first, to find the best matched output. As a result, the larger the corpus is, the more it will take time to generate responses.

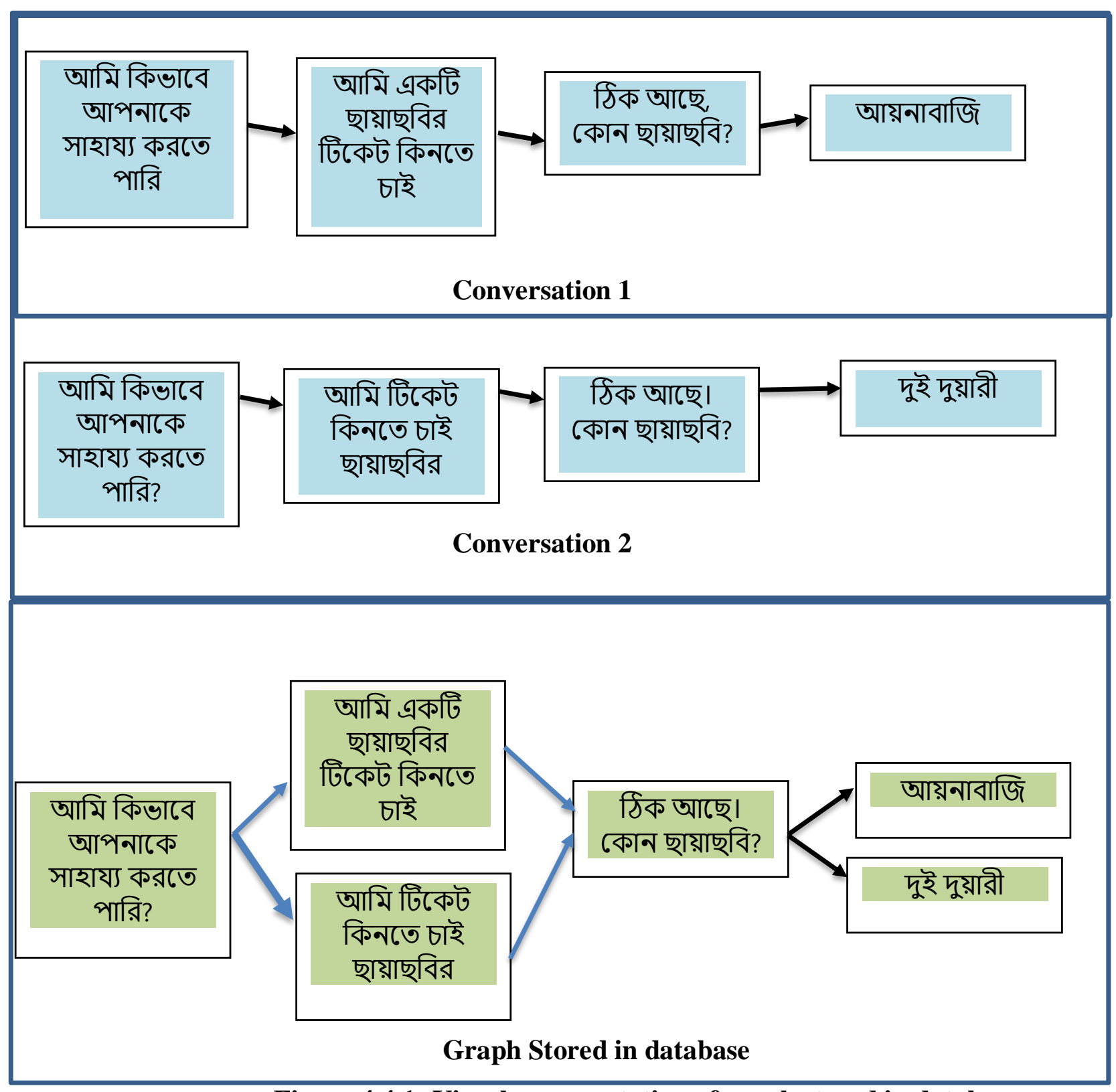

Figure 4.4 1: Visual representation of graph stored in database

Fig. 4.41 demonstrates how conversations are stored in database after training. 
For every conversation, there is a unique id generated. However, there are cases, where one question may have different replies in different conversations. This increases id numbers and results poor performance. This situations is tackled by graph approach of trained model. The different answers of similar questions are listed in the same conversation in the answer sections of the question. This results lesser number of id and increases performance of generating replies.

\subsubsection{ChatterBot}

Based on machine learning, ChatterBot is a conversational dialog engine powered by Python which is capable of giving responses based on a knowledge base. We choose this engine because it is language independent. Since Chatterbot has no language dependency in its design, so it is allowed to be trained to speak any language. It is a Python library that makes it easy to generate automated responses to a user's input for the creation of chatbot in any

language. To produce different types of responses, ChatterBot applies a selection of machine learning algorithms. This very feature makes it easy for developers to create chatbots and automate conversations with users.

The main class of the chatbot is a connecting point between each of ChatterBot's adapters. In this class, an input statement is returned from the input adapter, processed and stored by the logic and storage adapters, and then passed to the output adapter to be returned to the user.

Additionally, the machine-learning nature of ChatterBot allows an agent instance to improve its own knowledge of possible responses as it interacts with humans and other sources of informative data. An untrained instance of ChatterBot starts off with no knowledge of how to communicate. Each time a user enters a statement, the library saves the text that they entered and the text that the statement was in response to. As ChatterBot receives more input the 
number of responses that it can reply and the accuracy of each response in relation to the input statement increase. The program selects the closest matching response by searching for the closest 5 matching known statement that matches the input, the chatbot then chooses a response from the selection of known responses to that statement.

\subsubsection{Generating Responses}

Since out robot is a retrieval based open domain chatbot its success lies on the pattern matching algorithm. The algorithm of our system is as follows [24]:

1. After taking input from the console or any API, it sends it to the processing unit.

2. In processing part of the system, there is mainly one logic adapters which is Best Match Logic Adapter that uses Jaccard Similarity Index. The input given by the users is matched with the existing queries in the database and the sentence which matches with the input is selected. For a selected query,

3. The step (1), (2) and (3) continues in a loop until the user exits console.

4. When the user gives an input, it is stored in the knowledge base as a new query. So with each interaction with the user, the knowledge base learns a new query or response.

5. Our system is able to provide a Bangla corpus from its conversation history. 


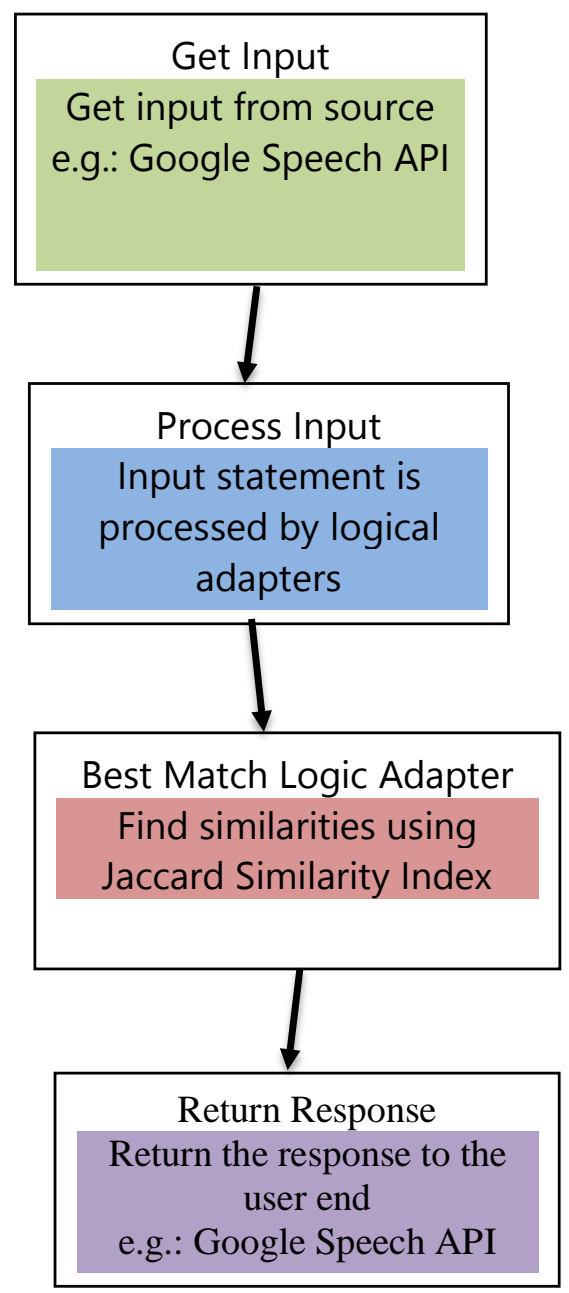

Figure 4.4.2 1: Working Flowchart of ChatterBot API [24]

After given a particular input statement, the input statement gets preprocessed before determining the response by logic adapter. In the preprocessor, any white space of the input statement is deleted. Afterwards, it removes any consecutive spaces from the input statement. Then the input statement is passed to the Match Best Logic adapter to generate answers. 


\subsubsection{Best Match Logic Adapter}

The best match logic adapter works by finding similarities between input and training data by using Jaccard Similarity Index. The Jaccard Similarity Index goes by,

$$
J(A, B)=\frac{|\mathrm{A} \cap \mathrm{B}|}{|\mathrm{A} \cup \mathrm{B}|}
$$

For example, our training dataset has an input question,

\section{“তোমাকে আমি মেরে ফেলবো”}

Now if we ask a question to the bot like, "তোমাকে মেরে ফেলা উচিত"

Then first the stop wards will be removed and we will come up with two sets,

$A=\{$ তোমাকে, আমি, মেরে $\}$

And B = \{ তোমাকে, মেরে, ফেলা $\}$

Now set $\mathrm{A}$ has 3 elements and $\mathrm{B}$ has 3 elements. The union of this sets is 4 and they have two common elements in them. So we get,

Jaccard Similarity Index $=2 \div 4=0.5$

According to our similarity threshold, this is considered to be a match. 


\section{CHAPTER V}

\section{RESULTS AND ANALYSIS}

After training our dataset, our bot becomes able to respond the question it gets input from the user to evaluate our model we decided to compare it with another chatbot.

\subsection{Comparison}

Since As we do not have Bangla chatbots to compare our model, we have compared our model with an English chatbot named Neuralconvo (http://neuralconvo.huggingface.co/) which is developed by deeplearning approach. We have asked five questions to both of the bot here is our findings.

We can see the result from the Figure 5.1 1. In the first question both of the system gave the same result. In second question Neuralconvo gave an answer which is bit ambiguous but makes sense, where as our model's reply is bit unusual. However, it is close to normal answers. Our 3rd question to our model was the 4th question of Neuralconvo. Neuralconvo's reply makes perfect sense. On the other hand our system's reply is a more intelligent than usual reply. Our next question was about life. In Neuralconvo the reply doesn't make any sense where as our reply is kind of usual answer though it was a bit ambiguous. The last question answer of Neuralconvo does not make any sense. However, our model gives a perfect reply to the last question. 


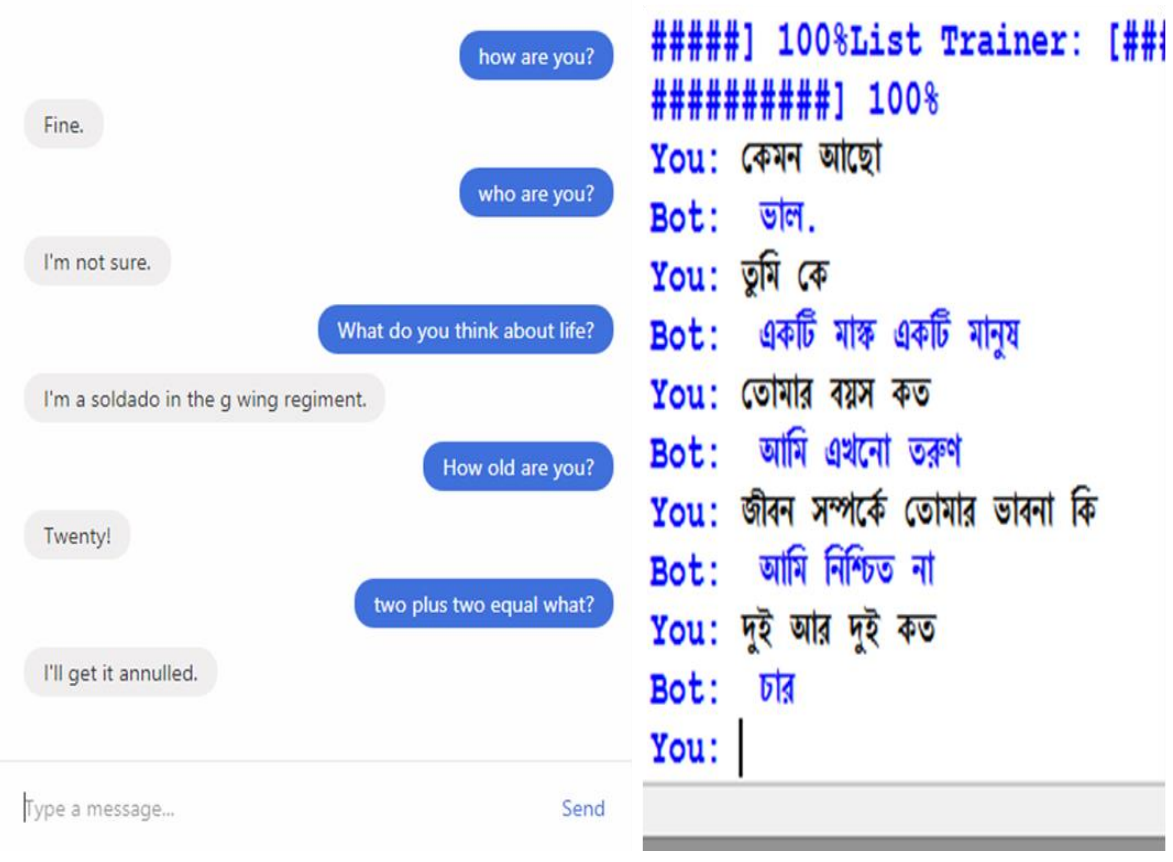

Figure 5.1 1: Comparison between a Chatbot \& the Virtual Bot

Neuralconvo is developed through the process of Deeplearning where as we used Chatterbot of python. In Deeplearning process there are some struggles with long sentences. On the other hand chatterbot gives reply by confidence value from trained dataset. In deep learning reply depends on fixed dataset. Our model keeps learning from the user input.

\subsection{Performance Efficiency}

Since we have executed our model in Raspberry-pi. Raspberry-pi is microcomputer. We have found different performance of virtual bot while we make different clock speed in CPU. The response time varies in different clock speed. In the Raspbian Linux distro the overclocking options on boot can be done by a software command running "sudo raspi-config" without voiding the 
warranty [28]. In those cases the Pi automatically shuts the overclocking down if the chip reaches $85^{\circ} \mathrm{C}\left(185^{\circ} \mathrm{F}\right)$,

\begin{tabular}{|l|l|l|}
\hline $\begin{array}{l}\text { CPU Speed } \\
\text { (arm } \\
\text { speed) } \\
\text { (CC/s) }\end{array}$ & $\begin{array}{l}\text { GPU Speed } \\
\text { (CC/s) }\end{array}$ & $\begin{array}{l}\text { Time Consumption } \\
\text { (in second) }\end{array}$ \\
\hline 800 & 126 & 5.5 \\
\hline 800 & 156 & 5.5 \\
\hline 1100 & 126 & 4 \\
\hline 1100 & 156 & 4 \\
\hline 1350 & 126 & 2.5 \\
\hline 1350 & 156 & 2.5 \\
\hline
\end{tabular}

Table 5.2 1: Comparison between CPU speed and time consumption

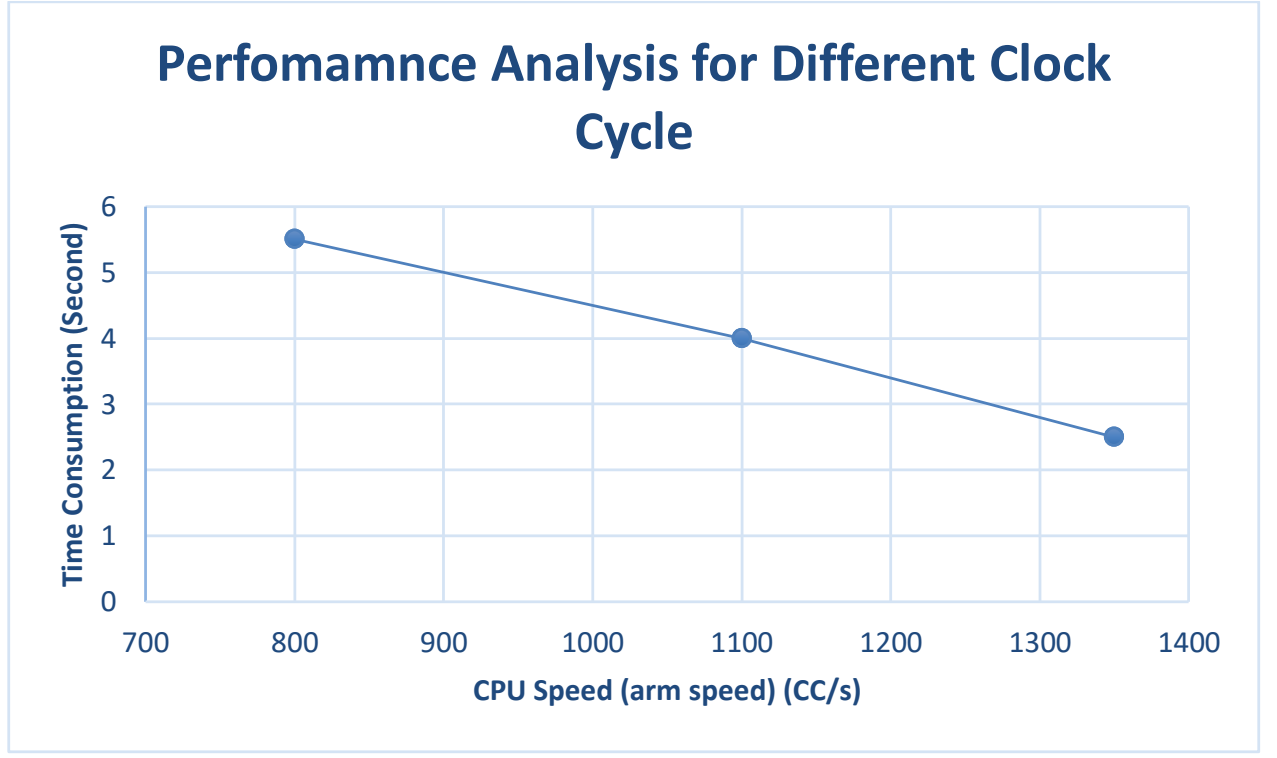

Figure 5.2 1: consumption time vs. CPU speed 
But it is possible to override automatic over-voltage and overclocking settings (voiding the warranty); an appropriately sized heat sink is needed to protect the chip from serious overheating. However, we have found GPU speed changes do not change our performance. As we know overclocking raspberry pi makes the pi really hot. Which is not good for long term. So, we find out that we can use threading. The Raspberry Pi 3 has a quad-core ARM Cortex-A53 processor. This was suggested to be highly dependent upon task threading and instruction set use. Benchmarks showed the Raspberry Pi 3 to be approximately $80 \%$ faster than the Raspberry Pi 2 in parallelized tasks [26].

\begin{tabular}{|c|c|}
\hline $\begin{array}{c}\text { CPU Speed (arm speed) } \\
(\mathrm{CC} / \mathrm{s})\end{array}$ & $\begin{array}{c}\text { Time Consumption } \\
\text { (in second) }\end{array}$ \\
\hline 800 & 4 \\
\hline 900 & 2.5 \\
\hline 1000 & 2 \\
\hline
\end{tabular}

Table 5.2 2: Time consumption after threading

Multithreading is a type of execution model that allows multiple threads to exist within the context of a process such that they execute independently but share their process resources. As we can see from the table, thread makes our system really fast. Overclocking is the process of tweaking motherboard settings to have the 'clock' run faster than the vendor certified. Can increase performance for single or multiple threaded scenarios. Also has risk because excess heat can be generated which can damage circuitry. So, a bit overclocking with multithreading gives best result of our system. 


\section{CHAPTER VI}

\section{CONCLUSION AND FUTURE WORK}

\subsection{Conclusion}

This research is a pioneering work in the field of dialogue system in Bengali. There are similar kind of bots which are based on English language. Due to lack of good resources in Bengali, good result did not come out on building frequent dialogue system. One of the main challenge was to build a reliable large dataset for training our model. We have refined our raw dataset through a number of processes. This processes let our system to be an open domain dialogue system. This bot also includes sentiment analysis of the question given by the user. Our bot will analysis the sentiment and react accordingly which is represent by graphical representation. The execution process of taking voice input, retrieving reply, analysis of sentiment and respond by voice with relevant facial expression will be conducted on a Raspberry-Pi. We think, our research will be helpful to build agent of any business system which needs response in Bangla. Moreover, our work will provide a Bengali conversation corpus which may help in the development of tools for Bengali Language Processing research.

\subsection{Future Work}

For future work, we dream to make it a fully functional social robot. We will try to give more actions which will give it an image more like human.Our dataset mostly based on Cornel Movie Dialogue Database which has been refined, translated and rechecked. However, we plan to make our very own dataset which will be based on Bengali literature work like novel, short story 
dialogues to train our model. This will make responses of our bot more intelligent and interesting. In our model we have used ChatterBot of python language which does not generate new responses. It only makes prediction of best response from dataset. In future we will implement GNN (generative neural network) which will generate new convenient responses. In our future work we have plan to build a robot like "SOFIA" in Bangla which will respond fluently in Bengali and express it's feelings[14]. As our model can work in closed domain too, we can build it to serve in specific business domain like hospital service, e-commerce etc. 


\section{REFERENCES}

[1] B. Duffy, "Anthropomorphism and the social robot", Robotics and Autonomous Systems, vol. 42, no. 3-4, pp. 177-190, 2003.

[2] A. Saenz, "Cleverbot Chat Engine Is Learning From The Internet To Talk Like A Human", Singularity Hub, 2018. [Online]. Available: https://singularityhub.com/2010/01/13/cleverbot-chat-engine-is-learningfrom-the-internet-to-talk-like-ahuman/\#sm.00000qca5mfaeaf21q7r826qj46b0. [Accessed: 24- Mar- 2018].

[3] D. Smith and F. Zeller (2017), The Death and Lives of hitchBOT: The Design and Implementation of a Hitchhiking Robot, Leonardo, vol. 50, no. 1, pp. 7778,2017

[4] Lichty P. (2018) The Aesthetics of Liminality: Augmentation as an Art Form. In: Geroimenko V. (eds) Augmented Reality Art. Springer Series on Cultural Computing. Springer, Cham

[5] T. Orin, "Implementation of a Bangla chatbot", Hdl.handle.net, 2017. [Online]. Available: http://hdl.handle.net/10361/8122. [Accessed: 14- Mar- 2018].

[6] Y. Nakauchi and R. Simmons, Autonomous Robots, vol. 12, no. 3, Y. Nakauchi and R. Simmons, Autonomous Robots, vol. 12, no. 3, pp. 313-324, 2002.

[7] A. TURING, "I.-COMPUTING MACHINERY AND INTELLIGENCE", Mind, vol., no. 236, pp. 433-460, 1950.

[8] E. Team, "What is Data Refining | Online Learning", Learn.geekinterview.com, 2018. [Online]. Available: http://www.learn.geekinterview.com/data-warehouse/data-quality/what-isdata-refining.html. [Accessed: 17- Mar- 2018].

[9] A. Raikov, Z. Avdeeva and A. Ermakov, "Big Data Refining on the Base of Cognitive Modeling", 2018

[10] "Implementing and Understanding Cosine Similarity", Masongallo.github.io, 2018. [Online]. Available: https://masongallo.github.io/machine/learning,/python/2016/07/29/cosinesimilarity.html. [Accessed: 17- Mar- 2018].

[11] "CosineDistance-Wolfram Language Documentation", Reference.wolfram.com, 2018. [Online]. Available: http://reference.wolfram.com/language/ref/CosineDistance.html. [Accessed: 17- Mar- 2018]

[12] "Introduction to Sets", Mathsisfun.com, 2018. [Online]. Available: 
https://www.mathsisfun.com/sets/sets-introduction.html. [Accessed: 17- Mar2018].

[13] S. leksikon, M. historie, M. logikk and K. Aubert, "mengdelære - Store norske leksikon", Store norske leksikon, 2018. [Online]. Available: https://snl.no/mengdel\%C3\%A6re. [Accessed: 24- Mar- 2018].

[14] "Vector Calculus: Understanding the Dot Product - BetterExplained", Betterexplained.com, 2018. [Online]. Available: https://betterexplained.com/articles/vector-calculus-understanding-the-dotproduct/. [Accessed: 17- Mar- 2018].

[15] D. Gershgorn, "Inside the mechanical brain of the world's first robot citizen", Quartz, 2018. [Online]. Available: https://qz.com/1121547/howsmart-is-the-first-robot-citizen/. [Accessed: 24- Mar- 2018].

[16] J. Weizenbaum, "ELIZA---a computer program for the study of natural language communication between man and machine", Communications of the $A C M$, vol. 9, no. 1, pp. 36-45, 1966.

[17] B. Setiaji and F. Wibowo, "Chatbot Using a Knowledge in Database: Human-to-Machine Conversation Modeling", 2016 7th International Conference on Intelligent Systems, Modelling and Simulation (ISMS), 2016.

[18] A. Das, S. Kottur, J. Moura, S. Lee and D. Batra, "Learning Cooperative Visual Dialog Agents with Deep Reinforcement Learning", 2017 IEEE International Conference on Computer Vision (ICCV), 2017.

[19] S. du Preez, M. Lall and S. Sinha, "An intelligent web-based voice chat bot", IEEE EUROCON 2009, 2009.

[20] O. Gambino, A. Augello, A. Caronia, G. Pilato, R. Pirrone and S. Gaglio, "Virtual conversation with a real talking head", 2008 Conference on Human System Interactions, 2008.

[21] "Raspberry Pi Documentation", Raspberrypi.org, 2018. [Online]. Available: https://www.raspberrypi.org/documentation/. [Accessed: 24- Mar2018].

[22] B. Vaidya, A. Patel, A. Panchal, R. Mehta, K. Mehta and P. Vaghasiya, "Smart home automation with a unique door monitoring system for old age people using Python, OpenCV, Android and Raspberry pi", 2017 International Conference on Intelligent Computing and Control Systems (ICICCS), 2017.

[23] N. Hossain, M. Kabir, T. Rahman, M. Hossen and F. Salauddin, "A realtime surveillance mini-rover based on OpenCV-Python-JAVA using Raspberry Pi 2", 2015 IEEE International Conference on Control System, Computing and Engineering (ICCSCE), 2015. 
[24] N. Kumar and R. Jatoth, "Development of cloud based light intensity monitoring system using raspberry $\mathrm{Pi} ", 2015$ International Conference on Industrial Instrumentation and Control (ICIC), 2015.
documentation", Chatterbot.readthedocs.io, 2018. [Online]. Available: https://chatterbot.readthedocs.io/en/stable/. [Accessed: 24-Mar- 2018].

[26] S. leksikon, M. historie, M. logikk and K. Aubert, "mengdelære - Store norske leksikon", Store norske leksikon, 2018. [Online]. Available: https://snl.no/mengdel\%C3\%A6re. [Accessed: 24- Mar- 2018].

[27] "What is Multithreading? - Definition from Techopedia", Techopedia.com, 2018. [Online]. Available: https://www.techopedia.com/definition/24297/multithreading-computerarchitecture. [Accessed: 24- Mar- 2018].

[28] "Introducing turbo mode: up to 50\% more performance for free Raspberry Pi", Raspberry Pi, 2018. [Online]. Available: https://www.raspberrypi.org/blog/introducing-turbo-mode-up-to-50-moreperformance-for-free/. [Accessed: 24- Mar- 2018].

[29] "Example for Learning a Naive Bayes Model | KNIME", Knime.com, 2018. [Online]. Available: https://www.knime.com/nodeguide/analytics/classification-and-predictivemodelling/example-for-learning-a-naive-bayes-model. [Accessed: 24- Mar2018]. 